\title{
Transient Hydraulic Pressure Fluctuation Analysis of a Hydroturbine via Ensemble Empirical Mode Decomposition
}

\author{
Canxun Du ${ }^{1, a}$, Weihua Gui ${ }^{1, b}$ Zhikun $\mathrm{Hu}^{2, \mathrm{c}}$ \\ ${ }^{1}$ School of Information Science and Engineering, Central South University, No.932 Lushannan Road, \\ Changsha 410083, Hunan Province, China \\ ${ }^{2}$ School of Physics and Electronics, Central South University, No.932 Lushannan Road, Changsha \\ 410083, Hunan Province, China \\ aemail: cx_du@chng.com.cn, bemail: gwh@csu.edu.cn, cemail: huzk@mail.csu.edu.cn
}

Keywords: Hilbert-Huang Transform, Ensemble Empirical Mode Decomposition, Time-frequency Analysis, Nonstationary Signal, Hydroturbine, Transient Process, Pressure Fluctuation, Draft Tube

\begin{abstract}
Hydroturbines are the key asset in hydroelectric power stations, and their monitoring and diagnosis play an important role in the asset management and maintenance. Hydraulic pressure fluctuation is inevitable during the operation of hydroturbines. It has nonstationarity during transient processes. The hydraulic pressure fluctuation signal measured in the draft tube of an axial-flow hydroturbine during a start-up transient process is analyzed based on ensemble empirical mode decomposition and Hilbert spectrum. The frequency components and their time evolution with the operating conditions are identified. A dominant low frequency component waxing and waning around a constant smaller than the rated rotating frequency exists throughout the transient process, indicating the possible occurrence of vortex core. Both the amplitude and the frequency are time varying, and are closely related to the operating conditions, especially the guide vane opening and switch, but they are not dominated by the speed. The closure and opening of guide vane and the on-off switching of hydroturbine can result in large amplitude pressure fluctuation, even the pressure surges or impulses in draft tube, which may excite the resonance of some parts and destroy the operating stability. The Hilbert spectrum based on ensemble empirical mode decomposition is suitable to analyze the nonstationary hydraulic pressure fluctuation signals of low frequency.
\end{abstract}

\section{Introduction}

The dynamic response of hydroturbines is a complicated coupling interaction of hydraulic fluctuation, mechanical vibration, and electromagnetic oscillation. Hydraulic force is the direct power source of hydroelectric sets, and water is the actuating medium, therefore hydraulic pressure fluctuation is inevitable during the operation of hydroturbines, and it is one of the main dynamic excitation sources [1-3].

Hydraulic pressure fluctuation in draft tube is a usual hydrodynamic phenomenon in hydroelectric power station. It is usually originated from the vortex core precession and unsteady flow in the draft tube, and can propagate towards both upstream and downstream. When a hydroturbine is operating at partial load, the unsteady flow may induce vortex rope precession in the draft tube, and thereby result in structural vibration of the hydroturbine, generator, and piping system, as well as electric oscillation in power network, and cloud noise. In the worst case, it may excite the resonance of some parts, induce power swing, and destroy the operating stability [1-3].

Some hydraulic pressure fluctuation phenomena in draft tube only occur under some specific operating conditions [1-3]. During transient processes, such as the start-up, shut-down, and load rejection or acceptance processes, the hydroturbine is modified from one operating point to another desired one. Namely, the operating condition parameters, such as the speed, power, and the guide vane opening, vary in a relatively wide range continuously. At some specific operating points during the transition of a hydroturbine, severe hydraulic pressure fluctuation, e.g. the pressure surge or impulse, may occur in the draft tube, which is very harmful to the operation stability. Therefore, it is 
very important to monitor and analyze the hydraulic pressure fluctuation in draft tube during the transient processes of hydroturbines.

Usually, the hydraulic pressure fluctuation in draft tube is nonstationary in terms of either amplitude or frequency, especially during the transient processes of hydroturbines [1-3]. It is more proper to use time-frequency analysis methods, rather than conventional signal processing methods like fast Fourier transform based on the assumption of signal stationarity, to identify the frequency components and to observe their time evolution, so as to extract the hydraulic pressure fluctuation features.

Some time-frequency analysis methods, such as short time Fourier transform, wavelet transform, and Cohen class distributions, have been applied to the signal analysis of conventional machinery. However, the inherent shortcomings limit their performance [4,5]: As linear transforms, both short time Fourier transform and wavelet transform are subject to Heisenberg uncertainty principle, i.e. the time localization and frequency resolution cannot be obtained at their highest simultaneously, either of them can only be enhanced at the expense of the other one, so that their time-frequency resolution is limited. In addition, as basis expansion based methods, the basis in either Fourier or wavelet transform is fixed, therefore they lack adaptability in simultaneously matching the complicated components inherent in signals. As a typical representative of Cohen class distributions, Wigner-Ville distribution has the best time-frequency resolution, but it is subject to the inevitable cross term interferences when applied to multiple component signals. This drawback causes it difficult to extract the true signal features in time-frequency domain. Various reduced interference distributions based on Wigner-Ville distribution may suppress the negative effect of cross terms, but at the expense of worse time-frequency resolution or suppress of auto terms.

Hilbert-Huang Transform (HHT) is a new method for nonlinear and nonstationary time series analysis. It extracts the intrinsic oscillation modes in signals by means of cubic spline interpolation, i.e. it does not assume any basis and thereby is adaptive in representing any signals. Moreover, it has fine time-frequency resolution, and is free of cross term interferences. Therefore it is effective in resolving the time varying structure of signal components accurately [6,7]. At present, it has been applied to data analysis in various domains, such as geophysics, biomedicine, finance, civil engineering, and mechanical engineering. However, the core algorithm of HHT, i.e. empirical mode decomposition (EMD), suffers from the mode mixing issue which is caused by singularities in signals. Fortunately, an improved method named ensemble empirical mode decomposition (EEMD) was proposed to tackle this issue [8].

Considering the complexity and nonstationarity of hydrodynamics during transient processes, HHT based on EEMD is used to analyze the on-site measured hydraulic pressure fluctuation signal in an axial-flow hydroturbine draft tube during start-up transient process. In section 2, HHT and EEMD are introduced briefly. In section 3, the hydraulic pressure fluctuation signal frequency components and their time evolution are identified successfully with EEMD and Hilbert spectrum. In section 4, some conclusions are drawn.

\section{Hilbert-Huang Transform and Ensemble Empirical Mode Decoposition}

Hilbert-Huang Transform is composed of two steps, i.e. Empirical Mode Decomposition (EMD) and signal representation by Hilbert Spectrum [6,7].

In the first step, a signal is decomposed into some Intrinsic Mode Functions (IMF) by means of EMD. IMF embodies the intrinsic oscillation mode embedded in the signal. It is defined to satisfy the requirements on mono-component signals, so as to compute instantaneous frequency by the local derivative of signal phase. EMD is essentially an iterative numerical approximation algorithm designed to extract IMF's from signals adaptively by cubic spline interpolation, according to the local characteristic time scale. Any signal can be approximated by a superposition of a series of IMF's.

Although EMD is well known and widely used, it suffers from a major drawback of mode mixing, which is often caused by intermittences in signals. In order to overcome this drawback, EEMD is proposed [8]. In EEMD, white noise of finite amplitude is added to the signal to provide a uniform 
reference frame in the time-frequency space, perturb the signal in the neighborhood of its true solutions, and thereby force the ensemble to exhaust all possible solutions in the EMD sifting process, and enable the signal components of different scales to collate in proper IMFs. In the ensemble mean of enough trials, the noise will be averaged out since it is different in separate trials. The detailed procedure of EEMD is as follows.

(1) Add a white noise series to the signal.

(2) Decompose the signal with added white noise into IMFs using traditional EMD.

(3) Return to step (1) and redo steps (1) and (2) for enough times predefined, but with different white noise series each time.

(4) Obtain the ensemble means of corresponding IMFs as the final result.

In the second step, Hilbert transform is applied to the IMF's, and their analytic signals are constructed, thereby both the instantaneous frequency and the instantaneous amplitude of each IMF can be calculated. Given the time, frequency and amplitude parameters, Hilbert amplitude/energy spectrum is constructed to represent the signal in joint time-frequency domain.

Different from conventional basis expansion based methods, such as short time Fourier transform and wavelet transform which employ basis functions of fixed form (e.g. sinusoidal/cosinoidal basis and wavelet basis respectively) to match the local structure of signals, HHT directly extracts the intrinsic fluctuation modes inherent in signals by means of numerical approximation, so it is adaptive in representing any signals and does not have any constraints on the way to tile the time-frequency plane.

HHT derives the instantaneous frequency from the local derivative of IMF phase using Hilbert transform which emphasizes the local properties of signals, so it has high time-frequency resolution. And also, Hilbert spectrum is a linear superposition of IMF time-frequency representations, rather than double integral involving quadratic terms of multiple components (e.g. Cohen class distributions), so it is free of cross term interferences.

In summary, HHT is highly adaptive, free of cross interferences, and has fine time-frequency resolution. These merits make it effective in analyzing the complicated and nonstationary hydraulic pressure fluctuation signals.

\section{Hydraulic Pressure Fluctuation Signal Analysis}

Hydroturbine Parameters and Measurement Configuration. The sketch of an axial-flow hydroturbine in a hydroelectric power station and the on-site pressure fluctuation measurement point configuration is shown in Fig. 1. The main parameters of the hydroturbine are listed in Table 1. The sampling frequency for collecting hydraulic pressure fluctuation signals and operating parameters is set to $56 \mathrm{~Hz}$.

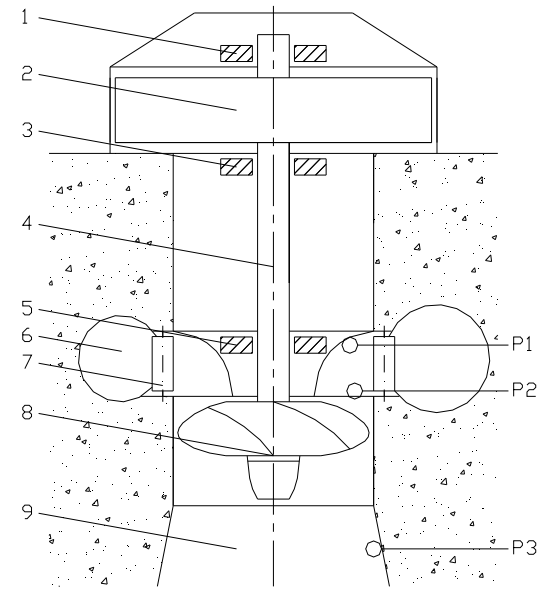

Fig. 1 Sketch for hydroturbine and measurement configuration

Hydroturbine schematic: 1 Upper guide bearing, 2 Generator rotor, 3 Thrust bearing, 4 Main shaft, 5 Turbine guide bearing, 6 Spiral case, 7 Guide vane, 8 Turbine runner, 9 Draft tube Measurement points configuration: P1 Beneath head cover, P2 At guide vane outlet, P3 At draft tube inlet 
Table 1 Hydroturbine parameters

\begin{tabular}{|c|c|c|}
\hline Model & Rated power & Rated speed \\
\hline ZD(F23)-LH-700 & $75 \mathrm{MW}$ & $88 \mathrm{revolution} / \mathrm{min}(1.467 \mathrm{~Hz})$ \\
\hline
\end{tabular}

Nonstationary Signal Analysis. A normal start-up process of the hydroturbine lasts for about 36 seconds. The hydraulic pressure fluctuation in the draft tube, the corresponding speed, power, guide vane opening, switch, excitation voltage and current, are shown in Fig. 2 and Fig. 3 respectively.

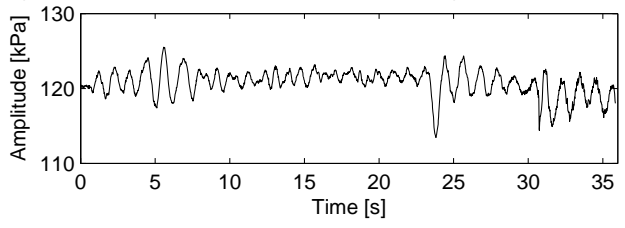

(a) Waveform

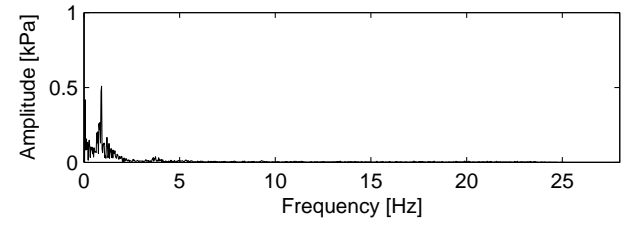

(b) Spectrum

Fig. 2 Hydraulic pressure fluctuation signal

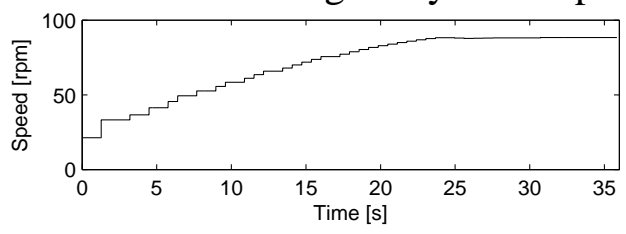

(a) Speed

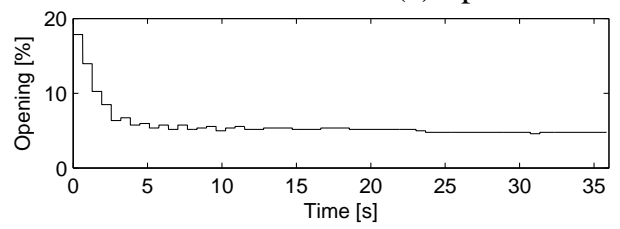

(c) Opening

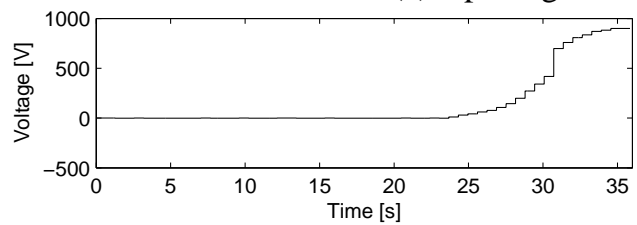

(e) Excitation voltage

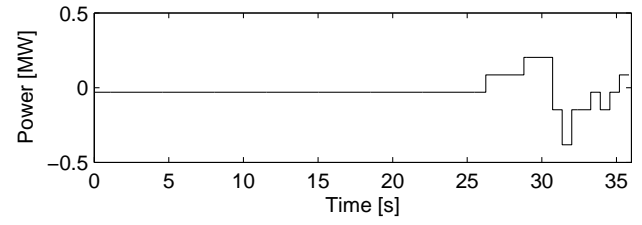

(b) Power

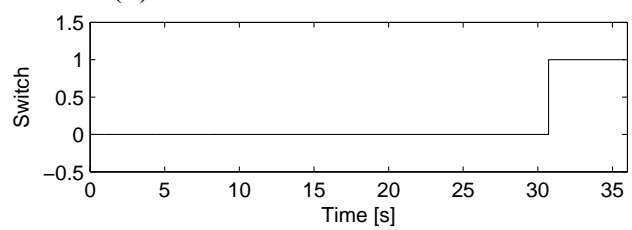

(d) Switch

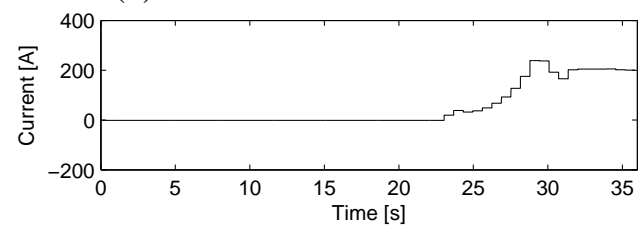

(f) Excitation current

Fig. 3 Operating parameters

From the waveform and the spectrum shown in Fig. 2, it can be seen that the pressure fluctuation signal in the start-up transient process is nonstationary, and the energy is concentrated in the low frequency band of $0-2 \mathrm{~Hz}$, with a peak around $1 \mathrm{~Hz}$.

In terms of the amplitude, the pressure fluctuation is closely related to the operating conditions. From the beginning to the instant of 10 second, while the power, switch, excitation voltage and current, keep unchanged, the speed increases gradually, and the guide vane opening decreases from $17 \%$ to $5 \%$ rapidly, so that the hydraulic pressure fluctuates in large amplitude. Subsequently, during the interval of 10-24 seconds, while the speed still increases, the other operating parameters remain nearly constant, as a result, the hydraulic pressure fluctuates in relatively small constant amplitude, indicating that it is not closely related to the speed. At the instant about 24 second, the speed reaches the rated one, and the hydroelectric power set begins to generate excitation voltage and current, so that the hydraulic pressure fluctuates in relatively large amplitude suddenly. From then on, till to the instant about 31 second, the excitation voltage and current increase gradually, and so does the power, but the other parameters keep unchanged. During this interval, the pressure fluctuation amplitude tends to decrease gradually. At the instant about 31 second, the hydroturbine is switched on to generate power. The power, together with the excitation voltage and current, exhibits significant change. Correspondingly the hydraulic pressure fluctuates in large amplitude suddenly. From then on, till to the end of the recorded start-up transient process, except for the power together with the excitation voltage and current, the other operating parameters keep stable at their rated ones, and the pressure fluctuation amplitude tends to be steady. 
The IMF's and residue obtained from EEMD is shown in Fig. 4. In the first two IMF's, significant impulses can be found at the instant about 31 second. It coincides with the event that the hydroturbine is switched on to generate power. Most energy of the signal is with the fourth IMF. From a global viewpoint, it oscillates at a period of about $1 \mathrm{~s}$, corresponding to the peak frequency of $1 \mathrm{~Hz}$ in the Fourier spectrum. From a local viewpoint, in the interval of $0-10 \mathrm{~s}$, when the guide vane opening decreases gradually, as well as in the interval of 24-36 s, when the power, together with the excitation voltage and current, vary in a large range, the pressure fluctuation appears like a nonstationary amplitude modulated process. In the interval of 10-24 s, when the speed continues increasing while the other parameters keep nearly constant, the hydraulic pressure fluctuates in relatively small amplitude. In a word, the pressure fluctuation amplitude is not sensitive to the speed, but closely related to the other operation parameters, such as the guide vane opening, power, and switch.
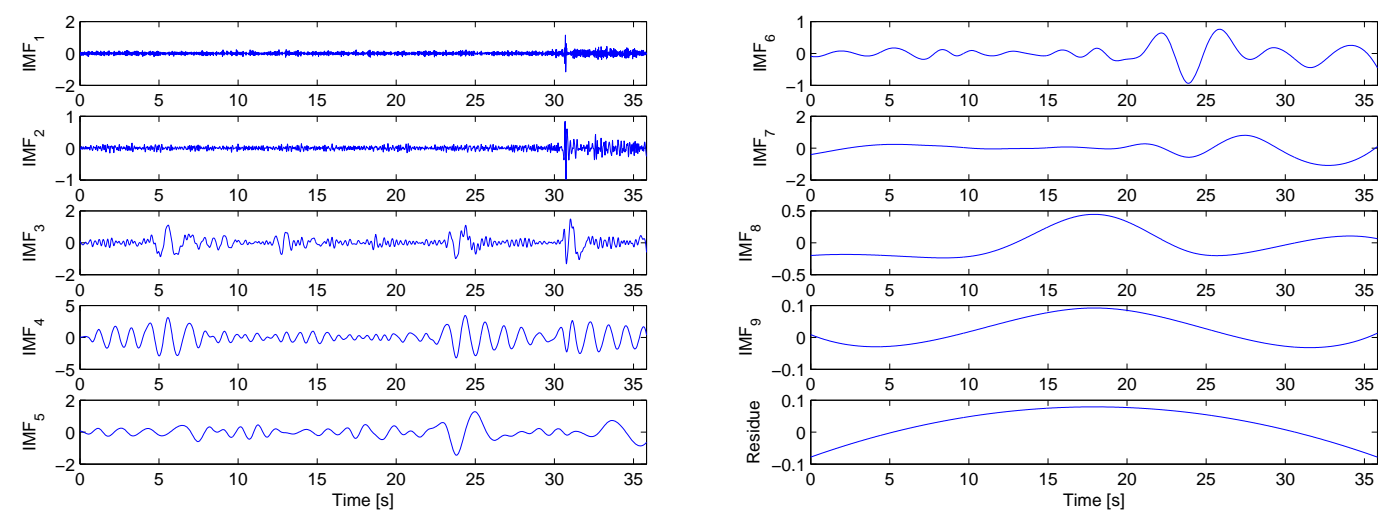

Fig. 4 EEMD result

The Hilbert energy spectrum is shown in Fig. 5. It can be noted that both the nonstationary pressure fluctuation of low frequency and the impulse due to switching the hydroturbine on to generate power are identified clearly. Although the speed increases from 0 to the rated one, the dominant instantaneous frequency of the pressure fluctuation appears to wax and wane around a constant of $1 \mathrm{~Hz}$, throughout the start-up transient process, rather than increases with the speed. This is far different from the common property of rotor vibration. The amplitude of the pressure fluctuation varies with the operating parameters, such as the speed, guide vane opening, power, and switch. At the instant about $6 \mathrm{~s}$, when the guide vane opening approaches the desired one, the Hilbert energy spectrum presents large amplitude. Although the draft tube is relatively far from the generator, the pressure fluctuation is likely to be related to the electromagnetic operation. At the instant about 24 $\mathrm{s}$, when the speed reaches the rated one, and the generator begins to generate excitation voltage and current, the Hilbert energy spectrum also presents relatively large amplitude. At the instant about $31 \mathrm{~s}$, the time-frequency distribution exhibits a significant impulse, which coincides with the event of switching the hydroturbine on to generate power.

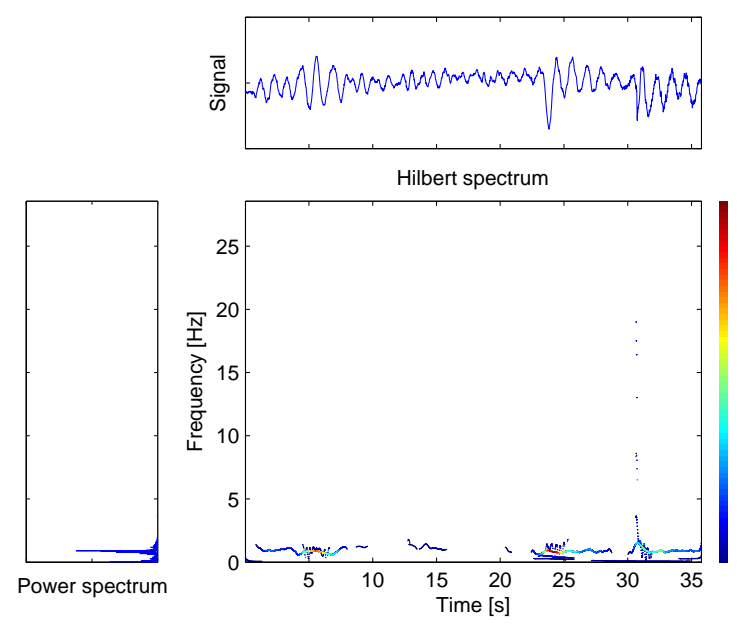

Fig. 5 Hilbert energy spectrum 


\section{Conclusions}

The pressure fluctuation in the draft tube is mainly composed of low frequency components and has strong nonstationarity. Although the rotating speed varies in a wide range during the transient process, the dominant frequency keeps waxing and waning around a constant smaller than the rotating frequency, rather than varies along with the rotating speed. This is far different from the characteristics of the rotor vibration. The fluctuation amplitude is time varying, and closely related to the operation conditions, especially the guide vane opening and switch. The closure and opening of the guide vane and the on-off switching of the hydroturbine can result in large amplitude pressure fluctuation, even the pressure impulses in the draft tube, which may influence the strength of some parts and destroy the operation stability.

Hilbert-Huang transform based on ensemble empirical mode decomposition is effective in resolving the low frequency components and the time varying features of the hydroturbine pressure fluctuation signal.

\section{References}

[1] H. Ohashi: Vibration and Oscillation of Hydraulic Machinery, Aldershot, Avebury Technical, England,UK (1991)

[2] Z. Ma, Y. Dong: Dynamics of Water Turbine Generator Set, Dalian, China: Dalian University of Technology Press (2003) (in Chinese)

[3] K. Wang: Vibration of Hydraulic Machinery, Beijing, China: Hydraulic and Electric Power Press (1986) (in Chinese)

[4] L. Cohen, Time-frequency distributions: A review, Proceedings of the IEEE, Vol. 77 (1989), p. 941

[5] F. Hlawatsch, G. F. Boudreaux-Bartels, Linear and quadratic time-frequency signal representations, IEEE Signal Processing Magazine, Vol. 9 (1992), p. 21

[6] N. E. Huang, Z. Shen, S. R. Long, et al, The empirical mode decomposition and the Hilbert spectrum for nonlinear and nonstationary time series analysis, Proceedings of the Royal Society of London, Series A, Vol. 454 (1998), p. 903

[7] N. E. Huang, S. P. Shen, Hilbert-Huang transform and its applications, Singapore: World Scientific (2005)

[8] Z. Wu, N. E. Huang, Ensemble empirical mode decomposition: a noise-assisted data analysis method, Advances in Adaptive Data Analysis, Vol. 1 (2009), p. 1 